\title{
Rigid Spacer-Mediated Synthesis of Bis-Spiroketal Ring Systems: Stereoselective Synthesis of Nonsymmetrical Spiro Disaccharides
}

\author{
Enrique M. Rubio, ${ }^{\dagger}$ M. Isabel García-Moreno, ${ }^{\ddagger}$ Patricia \\ Balbuena, ${ }^{\ddagger}$ Carmen Ortiz Mellet, ${ }^{,, \ddagger, \S}$ and José M. García \\ Fernández ${ }^{*} \dagger$
}

\section{Supporting Information}

General Methods. All solvents and reagents were purchased from commercial sources and used without further purification, except for dichloromethane, which was distilled under Ar stream over $\mathrm{CaH}_{2}$. 1,2-O-Isopropylidene- $\beta$-D-fructofuranose (5) was prepared according to described procedures (see ref. 9 in the manuscript). Optical rotations were measured at $20^{\circ} \mathrm{C}$ in $1-\mathrm{cm}$ or $1-\mathrm{dm}$ tubes on a Perkin-Elmer $141 \mathrm{MC}$ polarimeter. ${ }^{1} \mathrm{H}$ (and ${ }^{13} \mathrm{C}$ NMR) spectra were recorded at 500 (125.7) MHz with a Bruker 500 DRX instrument, respectively. 2D COSY, 1D TOCSY, HMQC and HSQC experiments were used to assist on NMR assignments. Thin-layer chromatography (TLC) was carried out on aluminium sheets coated with Kieselgel 60 F254 (E. Merck), with visualisation by UV light and by charring with $10 \% \mathrm{H}_{2} \mathrm{SO}_{4}$. Column chromatography was carried out on Silica Gel 60 (E. Merck, 230-400 mesh). FAB mass spectra were obtained with a Kratos MS-80 RFA instrument. The operating conditions were the following: the primary beam consisted of Xe atoms with a maximum energy of $8 \mathrm{keV}$; the samples were dissolved in thioglycerol, and the positive ions were separated and accelerated over a potential of $7 \mathrm{keV}$; NaI was added as cationizing agent. Elemental analyses were performed at the Instituto de Investigaciones Químicas (Sevilla, Spain).

3-O-(2-Bromomethylbenzyl)-1,2:4,5-di- $O$-isopropylidene- $\beta$-D-fructopyranose (7). To a solution of 1,2-dibromomethylbencene 6 (1.99 g, $7.56 \mathrm{mmol})$ in dry DMF (50 mL), NaH (60\% in mineral oil, $378 \mathrm{mg}, 9.45 \mathrm{mmol}$ ) was added and the suspension was stirred at room temperature for $15 \mathrm{~min}$. 1,2:4,5-Di- $O$-isopropylidene- $\beta$-D-fructopyranose 5 ( $1.0 \mathrm{~g}, 3.78 \mathrm{mmol})$ was then added and the reaction mixture was further stirred for $24 \mathrm{~h} . \mathrm{Et}_{2} \mathrm{O}(15 \mathrm{~mL})$ and water $(15 \mathrm{~mL})$ were added, the organic layer was separated, washed with water $(5 \times 10 \mathrm{~mL})$, dried $\left(\mathrm{MgSO}_{4}\right)$, filtered, and 
concentrated. The resulting residue was purified by column chromatography (1:8 EtOAc-petroleum ether) to yield 7 (628 mg, 73\%); $R_{f}=0.73\left(2: 5\right.$ EtOAc-petroleum ether); $[\alpha]_{\mathrm{D}}=-38.6(c 0.8$, $\left.\mathrm{CHCl}_{3}\right) ;{ }^{1} \mathrm{H}$ NMR $\left(500 \mathrm{MHz}, \mathrm{CDCl}_{3}\right): \delta=7.28-7.24(\mathrm{~m}, 4 \mathrm{H}, \mathrm{Ph}), 5.08,4.76\left(2 \mathrm{~d}, 2 \mathrm{H},{ }^{2} J_{\mathrm{H}, \mathrm{H}}=12.0\right.$ $\mathrm{Hz}, \mathrm{CHPh}), 4.73,4.58\left(2 \mathrm{~d}, 2 \mathrm{H},{ }^{2} J_{\mathrm{H}, \mathrm{H}}=10.2 \mathrm{~Hz}, \mathrm{CHBr}\right), 4.36\left(\mathrm{dd}, 1 \mathrm{H}, J_{3,4}=7.2 \mathrm{~Hz}, J_{4,5}=5.6 \mathrm{~Hz}\right.$, H-4), 4.21 (dd, 1 H, J5,6a $=2.5$ Hz, H-5), 3.96 (dd, 1 H, J6a,6b $=13.4$ Hz, H-6a), 3.99 (d, 1 H, H-6b), $3.96\left(\mathrm{~d}, 1 \mathrm{H}, J_{1 \mathrm{a}, 1 \mathrm{~b}}=8.8 \mathrm{~Hz}, \mathrm{H}-1 \mathrm{a}\right), 3.88$ (d, $\left.1 \mathrm{H}, \mathrm{H}-1 \mathrm{~b}\right), 3.53$ (d, $\left.1 \mathrm{H}, \mathrm{H}-3\right), 1.60,1.55,1.37,1.36$ (4 s, $\left.12 \mathrm{H}, \mathrm{CMe}_{2}\right) ;{ }^{13} \mathrm{C} \mathrm{NMR}\left(125.7 \mathrm{MHz}, \mathrm{CDCl}_{3}\right): \delta=136.8-128.5(\mathrm{Ph}), 112.1,109.1\left(\mathrm{CMe}_{2}\right), 104.3$ (C-2), 77.5 (C-4), 76.8 (C-3), 73.8 (C-5), 72.2 (C-1), $70.8\left(\mathrm{CH}_{2} \mathrm{Ph}\right), 60.4$ (C-6), $31.1\left(\mathrm{CH}_{2} \mathrm{Br}\right), 28.2$, 26.6, 26.3, $26.2\left(\mathrm{CMe}_{2}\right)$; FABMS: $m / z$ 465, $\left.467(80 \% \text {, [M+Na }]^{+}\right)$. Anal. Calcd for $\mathrm{C}_{20} \mathrm{H}_{27} \mathrm{BrO}_{6}: \mathrm{C}$ 54.18, H 6.14. Found: C 54.08, H 6.30.

\section{3-O-(2-Bromomethylbenzyl)-1,2- $O$-isopropylidene- $\beta$-D-fructopyranose (8) Compound 7} $(628 \mathrm{mg}, 1.41 \mathrm{mmol})$ was dissolved in $60 \%$ aqueous acetic acid $(3.5 \mathrm{~mL})$ and stirred at $45^{\circ} \mathrm{C}$ for 2 $\mathrm{h}$ The reaction mixture was then diluted with water $(5 \mathrm{~mL})$ and extracted with EtOAc $(4 \times 4 \mathrm{~mL})$. The combined organic phase was washed with saturated aqueous $\mathrm{NaHCO}_{3}(6 \mathrm{~mL})$, dried $\left(\mathrm{MgSO}_{4}\right)$, filtered, and concentrated. The resulting residue was purified by column chromatography $(2: 3 \rightarrow 1: 1$ EtOAc-petroleum ether) to give 8 (400 mg, 70\%); $R_{f}=0.47$ (3:1 EtOAc-petroleum ether); $[\alpha]_{\mathrm{D}}=-$ 60.3 (c 0.8 in $\left.\mathrm{CHCl}_{3}\right) ;{ }^{1} \mathrm{H}$ NMR (500 MHz, $\left.\mathrm{CD}_{3} \mathrm{OD}\right): \delta=7.37-7.26$ (m, $\left.4 \mathrm{H}, \mathrm{Ph}\right), 5.15,4.75(2 \mathrm{~d}, 2$ $\left.\mathrm{H},{ }^{2} J_{\mathrm{H}, \mathrm{H}}=11.6 \mathrm{~Hz}, \mathrm{CHPh}\right), 4.90,4.66\left(2 \mathrm{~d}, 2 \mathrm{H},{ }^{2} J_{\mathrm{H}, \mathrm{H}}=10.2 \mathrm{~Hz}, \mathrm{CHBr}\right), 3.93\left(\mathrm{dd}, 1 \mathrm{H}, J_{3,4}=9.8\right.$ $\left.\mathrm{Hz}, J_{4,5}=3.5 \mathrm{~Hz}, \mathrm{H}-4\right), 3.89\left(\mathrm{dd}, 1 \mathrm{H}, J_{6 \mathrm{a}, 6 \mathrm{~b}}=12.4 \mathrm{~Hz}, J_{5,6 \mathrm{a}}=1.4 \mathrm{~Hz}, \mathrm{H}-6 \mathrm{a}\right), 3.85\left(\mathrm{~d}, 1 \mathrm{H}, J_{1 \mathrm{a}, 1 \mathrm{~b}}=\right.$ $8.6 \mathrm{~Hz}, \mathrm{H}-1 \mathrm{a}), 3.84$ (m, 1 H, H-5), 3.80 (d, 1 H, H-1b), 3.69 (d, 1 H, H-3), 3.62 (d, 1 H, J5,6b = 1.9 $\mathrm{Hz}, \mathrm{H}-6 \mathrm{~b}), 1.39,1.30$ (2 s, $\left.6 \mathrm{H}, \mathrm{CMe}_{2}\right) ;{ }^{13} \mathrm{C} \mathrm{NMR}\left(125.7 \mathrm{MHz}, \mathrm{CD}_{3} \mathrm{OD}\right): \delta=137.1-128.1(\mathrm{Ph})$, $111.5\left(\mathrm{CMe}_{2}\right), 105.8$ (C-2), $75.8(\mathrm{C}-3), 71.8\left(\mathrm{CH}_{2} \mathrm{Ph}\right), 71.7$ (C-1), 71.5 (C-4), 70.1 (C-5), $64.3(\mathrm{C}-$ 6), $30.5\left(\mathrm{CH}_{2} \mathrm{Br}\right), 25.7,25.2\left(\mathrm{CMe}_{2}\right)$; FABMS: $m / z$ 425, $\left.427(70 \% \text {, [M+Na }]^{+}\right)$. Anal. Calcd for $\mathrm{C}_{17} \mathrm{H}_{23} \mathrm{BrO}_{6}$ : C 50.63, H 5.75. Found: C 50.23, H 5.79.

3-O-(2-Cloromethylbenzyl)-1,2:4,5-di- $O$-isopropylidene- $\beta$-D-fructopyranose (9). To a solution of the bromomethylbenzyl derivative $7(200 \mathrm{mg}, 0.34 \mathrm{mmol})$ in DMF (30 mL), saturated aqueous $\mathrm{NaCl}(4.0 \mathrm{~mL})$ was added and the mixture was stirred at $60^{\circ} \mathrm{C}$ for $16 \mathrm{~h}$. Water $(25 \mathrm{~mL})$ was added and the resulting solution was extracted with $\mathrm{Et}_{2} \mathrm{O}(5 \times 10 \mathrm{~mL})$. The combined organic layer was dried $\left(\mathrm{MgSO}_{4}\right)$, filtered, and concentrated to yield $9(170 \mathrm{mg}, 95 \%) ; R_{f}=0.73(2: 5$ EtOAc-petroleum ether); $[\alpha]_{\mathrm{D}}=-58.8\left(c 0.9, \mathrm{CHCl}_{3}\right) ;{ }^{1} \mathrm{H} \mathrm{NMR}\left(500 \mathrm{MHz}, \mathrm{CDCl}_{3}\right): \delta=7.39-7.26$ (m, $4 \mathrm{H}, \mathrm{Ph}), 5.11,4.76\left(\mathrm{~d}, 1 \mathrm{H},{ }^{2} J_{\mathrm{H}, \mathrm{H}}=12.0 \mathrm{~Hz}, \mathrm{CHPh}\right), 4.84,4.70\left(\mathrm{~d}, 1 \mathrm{H},{ }^{2} J_{\mathrm{H}, \mathrm{H}}=11.6 \mathrm{~Hz}, \mathrm{CHCl}\right)$, $4.38\left(\mathrm{dd}, 1 \mathrm{H}, J_{3,4}=7.3 \mathrm{~Hz}, J_{4,5}=5.4 \mathrm{~Hz}, \mathrm{H}-4\right), 4.22\left(\mathrm{dd}, 1 \mathrm{H}, J_{5,6 \mathrm{a}}=2.3 \mathrm{~Hz}, \mathrm{H}-5\right), 4.14$ (dd, $1 \mathrm{H}$, $\left.J_{6 \mathrm{a}, 6 \mathrm{~b}}=13.4 \mathrm{~Hz}, \mathrm{H}-6 \mathrm{a}\right), 4.01$ (d, 1 H, H-6b), 3.97 (d, 1 H, $\left.J_{1 \mathrm{a}, 1 \mathrm{~b}}=8.6 \mathrm{~Hz}, \mathrm{H}-1 \mathrm{a}\right), 3.89$ (d, $\left.1 \mathrm{H}, \mathrm{H}-1 \mathrm{~b}\right)$, 
$3.53(\mathrm{~d}, 1 \mathrm{H}, \mathrm{H}-3), 1.58,1.54,1.39,1.38\left(4 \mathrm{~s}, 12 \mathrm{H}, \mathrm{CMe}_{2}\right) ;{ }^{13} \mathrm{C} \mathrm{NMR}\left(125.7 \mathrm{MHz}, \mathrm{CDCl}_{3}\right): \delta=$ 136.6-128.4 (Ph), 112.1, $109.1\left(\mathrm{CMe}_{2}\right), 104.0$ (C-2), 77.5 (C-4), 76.7 (C-3), 73.8 (C-5), 72.2 (C-1), $70.8\left(\mathrm{CH}_{2} \mathrm{Ph}\right), 60.4(\mathrm{C}-6), 43.7\left(\mathrm{CH}_{2} \mathrm{Cl}\right), 28.2,26.6,26.3,26.2\left(\mathrm{CMe}_{2}\right)$; FABMS: $m / z$ 421, 423 $\left(50 \%,[\mathrm{M}+\mathrm{Na}]^{+}\right)$; elemental analysis calcd. (\%) for $\mathrm{C}_{20} \mathrm{H}_{27} \mathrm{ClO}_{6}: \mathrm{C} 60.22, \mathrm{H} \mathrm{6.82}$; found: $\mathrm{C} 60.28, \mathrm{H}$ 6.56 .

3-O-(2-Cloromethylbenzyl)-1,2-O-isopropylidene- $\beta$-D-fructopyranose (10). Treatment of 9 (150 mg, $0.37 \mathrm{mmol}$ ) with 60\% aqueous $\mathrm{AcOH}$, following the procedure above described for the preparation of 8 , and purification by column chromatography $(2: 3 \rightarrow 1: 1$ EtOAc-petroleum ether) yielded $10(120 \mathrm{mg}, 88 \%) ; R_{f}=0.47(3: 1 \mathrm{EtOAc} /$ petroleum ether $) ;[\alpha]_{\mathrm{D}}=-79.8\left(c 1.6, \mathrm{CHCl}_{3}\right) ;{ }^{1} \mathrm{H}$ NMR (500 MHz, $\left.\mathrm{CD}_{3} \mathrm{OD}\right): \delta=7.38-7.28(\mathrm{~m}, 4 \mathrm{H}, \mathrm{Ph}), 5.16,4.74\left(\mathrm{~d}, 1 \mathrm{H},{ }^{2} J_{\mathrm{H}, \mathrm{H}}=11.6 \mathrm{~Hz}, \mathrm{CHPh}\right)$, 4.88, $4.86\left(\mathrm{~d}, 1 \mathrm{H},{ }^{2} J_{\mathrm{H}, \mathrm{H}}=11.8 \mathrm{~Hz}, \mathrm{CHCl}\right), 3.92\left(\mathrm{dd}, 1 \mathrm{H}, J_{3,4}=9.8 \mathrm{~Hz}, J_{4,5}=3.4 \mathrm{~Hz}, \mathrm{H}-4\right), 3.89$ (dd, $\left.1 \mathrm{H}, J_{6 \mathrm{a}, 6 \mathrm{~b}}=12.4 \mathrm{~Hz}, J_{5,6 \mathrm{a}}=1.4 \mathrm{~Hz}, \mathrm{H}-6 \mathrm{a}\right), 3.83\left(\mathrm{~d}, 1 \mathrm{H}, J_{1 \mathrm{a}, 1 \mathrm{~b}}=8.6 \mathrm{~Hz}, \mathrm{H}-1 \mathrm{a}\right), 3.82(\mathrm{~m}, 1 \mathrm{H}, \mathrm{H}-5)$, 3.79 (d, $1 \mathrm{H}, \mathrm{H}-1 \mathrm{~b}), 3.68$ (d, $1 \mathrm{H}, \mathrm{H}-3), 3.62$ (d, $\left.1 \mathrm{H}, J_{5,6 \mathrm{~b}}=1.9 \mathrm{~Hz}, \mathrm{H}-6 \mathrm{~b}\right), 1.39,1.30(2 \mathrm{~s}, 6 \mathrm{H}$, $\left.\mathrm{CMe}_{2}\right) ;{ }^{13} \mathrm{C} \mathrm{NMR}\left(125.7 \mathrm{MHz}, \mathrm{CD}_{3} \mathrm{OD}\right): \delta=129.8-128.0(\mathrm{Ph}), 111.5\left(\mathrm{CMe}_{2}\right), 105.8(\mathrm{C}-2), 75.8(\mathrm{C}-$ 3), $71.9\left(\mathrm{CH}_{2} \mathrm{Ph}\right), 71.7(\mathrm{C}-1), 71.5(\mathrm{C}-4), 70.1(\mathrm{C}-5), 64.3(\mathrm{C}-6), 43.1\left(\mathrm{CH}_{2} \mathrm{Cl}\right), 25.7,25.2\left(\mathrm{CMe}_{2}\right)$; FABMS: $m / z 381(60 \% \text {, [M+Na] }]^{+}$. Anal. Calcd for $\mathrm{C}_{17} \mathrm{H}_{23} \mathrm{ClO}_{6}$ : C 56.90, H 6.46. Found: C 56.58, H 6.33 .

4,5-Di-O-Benzyl-3- $O$-(2-bromomethylbenzyl)-1,2- $O$-isopropylidene- $\beta$-D-fructopyranose (11). To an suspension of $\mathrm{NaH}(60 \%$ in mineral oil, $66 \mathrm{mg}, 1.6 \mathrm{mmol})$ and benzyl bromide (794 $\mu \mathrm{L}, 6.68 \mathrm{mmol})$ in DMF $(7 \mathrm{~mL})$, a solution of $10(120 \mathrm{mg}, 0.33 \mathrm{mmol})$ in dry DMF $(3 \mathrm{~mL})$ was dropwise added. The reaction mixture was stirred for $2 \mathrm{~h}$ at $50{ }^{\circ} \mathrm{C}$, then quenched with $\mathrm{H}_{2} \mathrm{O}(2 \mathrm{~mL})$, extracted with $\mathrm{Et}_{2} \mathrm{O}(5 \times 5 \mathrm{~mL})$, the combined organic layer was dried $\left(\mathrm{MgSO}_{4}\right)$ and concentrated. The resulting residue was purified by column chromatography (1:5 EtOAc-petroleum ether) to give $11(122 \mathrm{mg}, 70 \%) ; \mathrm{R}_{f}=0.78$ (1:4 EtOAc-petroleum ether); $[\alpha]_{\mathrm{D}}=-37.1\left(c 3.8\right.$ in $\left.\mathrm{CHCl}_{3}\right) ;{ }^{1} \mathrm{H} \mathrm{NMR}$ $\left(500 \mathrm{MHz}_{\mathrm{CDCl}}\right): \delta=7.39-7.24(\mathrm{~m}, 14 \mathrm{H}, \mathrm{Ph}), 5.16,4.81\left(2 \mathrm{~d}, 2 \mathrm{H},{ }^{2} J_{\mathrm{H}, \mathrm{H}}=12.0 \mathrm{~Hz}, \mathrm{CHPh}\right), 4.75$, $4.68\left(2 \mathrm{~d}, 2 \mathrm{H},{ }^{2} J_{\mathrm{H}, \mathrm{H}}=12.5 \mathrm{~Hz}, \mathrm{CHPh}\right), 4.62,4.59\left(2 \mathrm{~d}, 2 \mathrm{H},{ }^{2} J_{\mathrm{H}, \mathrm{H}}=11.5 \mathrm{~Hz}, \mathrm{CHPh}\right), 4.59(\mathrm{~s}, 2 \mathrm{H}$, $\left.\mathrm{CH}_{2} \mathrm{Br}\right), 3.96\left(\mathrm{~d}, 1 \mathrm{H}, J_{3,4}=9.8 \mathrm{~Hz}, \mathrm{H}-3\right), 3.93\left(\mathrm{~d}, 1 \mathrm{H}, J_{1 \mathrm{a}, 1 \mathrm{~b}}=8.6 \mathrm{~Hz}, \mathrm{H}-1 \mathrm{a}\right), 3.91$ (dd, $1 \mathrm{H}, J_{4,5}=$ $3.4 \mathrm{~Hz}, \mathrm{H}-4), 3.90$ (d, $1 \mathrm{H}, \mathrm{H}-1 \mathrm{~b}), 3.81$ (dd, 1 H, $\left.J_{6 \mathrm{a}, 6 \mathrm{~b}}=13.0 \mathrm{~Hz}, J_{5,6 \mathrm{a}}=2.0 \mathrm{~Hz}, \mathrm{H}-6 \mathrm{a}\right), 3.80$ (m, $1 \mathrm{H}$, $\mathrm{H}-5), 3.76\left(\mathrm{dd}, 1 \mathrm{H}, J_{5,6 \mathrm{~b}}=1.5 \mathrm{~Hz}, \mathrm{H}-6 \mathrm{~b}\right), 1.45,1.41\left(2 \mathrm{~s}, 6 \mathrm{H}, \mathrm{CMe}_{2}\right) ;{ }^{13} \mathrm{C} \mathrm{NMR}(125.7 \mathrm{MHz}$, $\left.\mathrm{CD}_{3} \mathrm{OD}\right): \delta=137.2-127.6(\mathrm{Ph}), 111.7\left(\mathrm{CMe}_{2}\right), 105.8(\mathrm{C}-2), 80.1(\mathrm{C}-4), 75.4(\mathrm{C}-3), 73.3(\mathrm{C}-5), 72.3$ $\left(\mathrm{CH}_{2} \mathrm{Ph}\right), 72.2(\mathrm{C}-1), 71.8,71.6\left(\mathrm{CH}_{2} \mathrm{Ph}\right), 61.5(\mathrm{C}-6), 31.2\left(\mathrm{CH}_{2} \mathrm{Br}\right), 26.7,26.4\left(\mathrm{CMe}_{2}\right)$; FABMS: $m / z$ 607, $605\left(20 \%,[\mathrm{M}+\mathrm{Na}]^{+}\right)$. Anal. Calcd for $\mathrm{C}_{31} \mathrm{H}_{35} \mathrm{BrO}_{6}: \mathrm{C} 63.81, \mathrm{H}$ 6.05. Found: $\mathrm{C} 63.74, \mathrm{H}$ 5.79 . 
4,5-Di-O-Benzyl-1,2- $O$-isopropylidene- $\beta$-D-fructopyranose 1,2:4,5-Di- $O$-isopropylidene$\boldsymbol{\beta}$-D-fructopyranose 3,3'-O-(o-Xylenylene) (12): To a solution of 5 (59 $\mathrm{mg}, 0.23 \mathrm{mmol})$ in dry DMF (2 mL), an oily suspension of $\mathrm{NaH}(60 \%, 19 \mathrm{mg}, 0.46 \mathrm{mmol}, 2.5 \mathrm{eq})$ was added and the reaction mixture was stirred at room temperature for $15 \mathrm{~min}$. A solution of $\mathbf{1 1}$ (100 $\mathrm{mg}, 0.186$ $\mathrm{mmol})$ in DMF (3 $\mathrm{mL})$ was then added and the reaction mixture was further stirred at room temperature for $1 \mathrm{~h}$. The reaction was quenched by addition of $\mathrm{MeOH}(2 \mathrm{~mL})$ and water $(4 \mathrm{~mL})$, and extracted with $\mathrm{Et}_{2} \mathrm{O}(2 \times 8 \mathrm{~mL})$. The combined organic layer was washed with water $(5 \times 5$ $\mathrm{mL})$, dried $\left(\mathrm{MgSO}_{4}\right)$, concentrated and purified by column chromatography (1:6 EtOAc-petroleum ether) to yield 12 (83 mg, 70\%); $R_{f}=0.44\left(1: 3 \mathrm{EtOAc} /\right.$ petroleum ether); $[\alpha]_{\mathrm{D}}=-65.3(c 0.7$, $\left.\mathrm{CHCl}_{3}\right) ;{ }^{1} \mathrm{H} \mathrm{NMR}\left(500 \mathrm{MHz}, \mathrm{CDCl}_{3}\right): \delta=7.31-7.24(\mathrm{~m}, 14 \mathrm{H}, \mathrm{Ph}), 5.15,4.78\left(2 \mathrm{~d}, 2 \mathrm{H},{ }^{2} J_{\mathrm{H}, \mathrm{H}}=\right.$ $12.5 \mathrm{~Hz}, \mathrm{CHPh}), 4.96,4.73\left(2 \mathrm{~d}, 2 \mathrm{H},{ }^{2} J_{\mathrm{H}, \mathrm{H}}=12.4 \mathrm{~Hz}, \mathrm{CHPh}\right), 4.67,4.59\left(2 \mathrm{~d}, 2 \mathrm{H},{ }^{2} J_{\mathrm{H}, \mathrm{H}}=12.5 \mathrm{~Hz}\right.$, $\mathrm{C} H \mathrm{Ph}), 4.33\left(\mathrm{dd}, J_{3,4}=7.2 \mathrm{~Hz}, J_{4,5}=5.7 \mathrm{~Hz}, 1 \mathrm{H}, \mathrm{H}-4\right), 4.18\left(\mathrm{dd}, 1 \mathrm{H}, J_{5,6 \mathrm{a}}=1.8 \mathrm{~Hz}, \mathrm{H}-5\right), 4.10(\mathrm{dd}$, $\left.1 \mathrm{H}, J_{6 \mathrm{a}, 6 \mathrm{~b}}=13.4 \mathrm{~Hz}, \mathrm{H}-6 \mathrm{a}\right), 3.96$ (d, 1 H, H-6b), 3.94 (d, 1 H, $J_{3^{\prime}, 4}$, = 9.8 Hz, H-3'), 3.90 (dd, 1 H, $\left.J_{4^{\prime}, 5^{\prime}}=10.0 \mathrm{~Hz}, J_{5^{\prime}, 6 \mathrm{a}^{\prime}}=2.9 \mathrm{~Hz}, \mathrm{H}-4^{\prime}\right), 3.88$ (d, $\left.1 \mathrm{H}, J_{1 \mathrm{a}^{\prime}, 1 \mathrm{~b}^{\prime}}=8.7 \mathrm{~Hz}, \mathrm{H}-1 \mathrm{a}^{\prime}\right), 3.85$ (d, $2 \mathrm{H}, J_{1 \mathrm{a}, 1 \mathrm{~b}}=8.5$ Hz, H-1b', H-1a), 3.79 (d, 1 H, $\left.{ }^{2} J_{\mathrm{H}, \mathrm{H}}=8.6 \mathrm{~Hz}, \mathrm{H}-1 \mathrm{~b}\right), 3.77$ (m, 2 H, H-5', H-6a'), 3.74 (dd, 1 H, $\left.J_{5}, 6 \mathrm{~b}^{\prime}=1.4 \mathrm{~Hz}, \mathrm{H}-6 \mathrm{~b}^{\prime}\right), 3.44$ (d, $\left.1 \mathrm{H}, \mathrm{H}-3\right), 1.51,1.45,1.44,1.41,1.37,1.33$ (6 s, $\left.18 \mathrm{H}, \mathrm{CMe}_{2}\right){ }^{13} \mathrm{C}$ NMR (125.7 MHz, $\left.\mathrm{CDCl}_{3}\right): \delta=138.4-127.3(\mathrm{Ph}), 112.1,111.7,109.0\left(\mathrm{CMe}_{2}\right), 105.9(\mathrm{C}-2$ ') 104.4 (C-2), 80.2 (C-4'), 77.7 (C-4), 76.4 (C-3), 75.1 (C-3'), 73.8 (C-5), 73.5 (C-5'), 72.2, $72.1\left(\mathrm{CH}_{2} \mathrm{Ph}\right)$, 71.9 (C-1, C-1'), 71.6, $70.5\left(\mathrm{CH}_{2} \mathrm{Ph}\right), 61.5$ (C-6'), 60.3 (C-6), 28.2, 26.9, 26.7, 26.4, $26.2\left(\mathrm{CMe}_{2}\right)$; FABMS: $m / z 785\left(100 \%,[\mathrm{M}+\mathrm{Na}]^{+}\right)$. Anal. Calcd for $\mathrm{C}_{43} \mathrm{H}_{54} \mathrm{O}_{12}: \mathrm{C} 67.70, \mathrm{H}$ 7.13. Found: C 67.45, H 6.87 .

\section{$\beta$-D-Fructofuranose 4,5-di- $O$-Benzyl- $\beta$-D-fructopyranose 1,2':2,1'-Dianhydride 3,3'- $O$ -}

(o-Xylylene) (13). To a solution of $12(124 \mathrm{mg}, 0.162 \mathrm{mmol})$ in $\mathrm{CH}_{2} \mathrm{Cl}_{2}(36 \mathrm{~mL})$ at $-78{ }^{\circ} \mathrm{C}$ under Ar, trifluoromethanesulfonic acid $(22 \mu \mathrm{L})$ was added. The reaction mixture was allowed to reach room temperature and stirred for $1 \mathrm{~h} . \mathrm{Et}_{3} \mathrm{~N}(1 \mathrm{~mL})$ was then added, the reaction mixture was concentrated and the resulting residue was purified column chromatography using 3:1 EtOAcpetroleum ether to give 13 (40 $\mathrm{mg}(41 \%) ; R_{f}=0.40$ (4:1 EtOAc-petroleum ether); $[\alpha]_{\mathrm{D}}=-122.9(c$ 1.15 in $\left.\mathrm{CHCl}_{3}\right) ;{ }^{1} \mathrm{H} \mathrm{NMR}\left(500 \mathrm{MHz}, \mathrm{CDCl}_{3}\right): \delta=7.40-7.12(\mathrm{~m}, 14 \mathrm{H}, \mathrm{Ph}), 5.69,5.58(2 \mathrm{~d}, 2 \mathrm{H}$, $\left.{ }^{2} J_{\mathrm{H}, \mathrm{H}}=8.1 \mathrm{~Hz}, \mathrm{CHPh}\right), 4.78,4.72\left(2 \mathrm{~d}, 2 \mathrm{H},{ }^{2} J_{\mathrm{H}, \mathrm{H}}=12.7 \mathrm{~Hz}, \mathrm{CHPh}\right), 4.55,4.50\left(2 \mathrm{~d}, 2 \mathrm{H},{ }^{2} J_{\mathrm{H}, \mathrm{H}}=\right.$ $12.1 \mathrm{~Hz}, \mathrm{CHPh}), 4.46,4.34\left(2 \mathrm{~d}, 2 \mathrm{H},{ }^{2} J_{\mathrm{H}, \mathrm{H}}=8.1 \mathrm{~Hz}, \mathrm{CHPh}\right), 4.40\left(\mathrm{t}, 1 \mathrm{H}, J_{3,4}=J_{4,5}=7.8 \mathrm{~Hz}, \mathrm{H}-4\right)$, $4.16\left(\mathrm{~d}, 1 \mathrm{H}, J_{1 \mathrm{a}, 1 \mathrm{~b}}=12.8 \mathrm{~Hz}, \mathrm{H}-1 \mathrm{a}\right), 3.95\left(\mathrm{~d}, 1 \mathrm{H}, J_{1 \mathrm{a}, 1 \mathrm{~b}}=12.9 \mathrm{~Hz}, \mathrm{H}-1 \mathrm{a}\right), 3.91$ (d, $\left.1 \mathrm{H}, \mathrm{H}-1 \mathrm{~b}\right), 3.90$ $\left(\mathrm{d}, 1 \mathrm{H}, J_{3^{\prime}, 4^{\prime}}=9.5 \mathrm{~Hz}, \mathrm{H}-3^{\prime}\right), 3.85$ (dd, $\left.1 \mathrm{H}, J_{5,6 \mathrm{~b}}=5.6 \mathrm{~Hz}, \mathrm{H}-5\right), 3.87$ (d, $\left.1 \mathrm{H}, \mathrm{H}-3\right), 3.82$ (d, $1 \mathrm{H}, \mathrm{H}-$ 1b), 3.75 (dd, 1 H, $J_{6 a^{\prime}, 6 b^{\prime}}=12.5$ Hz, $J_{5^{\prime}, 6 a^{\prime}}=2.1$ Hz, H-6a'), 3.74 (m, 1 H, H-5'), 3.69 (m, 1 H, H- 
6a), 3.66 (d, 1 H, H-6b), 3.61 (dd, 1 H, $J_{4}, 5^{5}=3.2 \mathrm{~Hz}, \mathrm{H}-4$ '), 3.59 (m, $\left.1 \mathrm{H}, \mathrm{H}-6 \mathrm{~b}\right) ;{ }^{13} \mathrm{C}$ NMR (125.7 $\mathrm{MHz}, \mathrm{CDCl}_{3}$ ): $\delta=138.6-127.3(\mathrm{Ph}), 101.3$ (C-2), 97.5 (C-2'), 89.6 (C-3), 80.3 (C-5), 80.0 (C-3'), 77.9 (C-4'), 74.7 (C-4), $73.1\left(\mathrm{CH}_{2} \mathrm{Ph}\right), 72.9$ (C-5'), 71.6, $71.4\left(\mathrm{CH}_{2} \mathrm{Ph}\right), 66.4(\mathrm{C}-1), 62.9$ (C-1), 62.5 (C-6), 61.0 (C-6'); FABMS: $\left.m / z 629(65 \% \text {, [M+Na] }]^{+}\right)$. Anal. Calcd for $\mathrm{C}_{34} \mathrm{H}_{38} \mathrm{O}_{10}$ : C 67.31, H 6.31 . Found: C, 67.51, H 6.25.

$\beta$-D-Fructofuranose $\beta$-D-Fructopyranose 1,2':2,1'-Dianhydride (1). Conventional catalytic hydrogenation of 13 (30 mg, $0.092 \mathrm{mmol}$ ) with 10\% Pd-C in 1:1 EtOAc-MeOH containing $10 \% \mathrm{HCOOH}(1 \mathrm{~mL})$ at $1 \mathrm{~atm}$ overnight, afforded the fully unprotected bis-spiro fructodisaccharide 1 having physicochemical and spectroscopic properties identical to those reported $\left([\alpha]_{\mathrm{D}}=-181(c\right.$ 1.2 in $\mathrm{H}_{2} \mathrm{O}$ ); Lit. $[\alpha]_{\mathrm{D}}=-179$ and -183 (c 3.6 and 1.8 in $\mathrm{H}_{2} \mathrm{O}$, respectively); see refs. 1 and 11 in the manuscript). The identity of $\mathbf{1}$ was additionally confirmed by GC after transformation into the corresponding hexa- $O$-trimethylsilyl derivative, following the protocol previously reported (see ref. 5 in the manuscript). 